\title{
Manuela Raccanello, Proust in Italia. Le traduzioni della "Recherche"
}

\section{Francesca Piselli}

\section{Q OpenEdition \\ 1 Journals}

\section{Edizione digitale}

URL: http://journals.openedition.org/studifrancesi/581

DOI: 10.4000/studifrancesi.581

ISSN: 2421-5856

\section{Editore}

Rosenberg \& Sellier

\section{Edizione cartacea}

Data di pubblicazione: 1 aprile 2015

Paginazione: 187-188

ISSN: 0039-2944

\section{Notizia bibliografica digitale}

Francesca Piselli, « Manuela Raccanello, Proust in Italia. Le traduzioni della "Recherche" », Studi Francesi [Online], 175 (LIX | I) | 2015, online dal 01 avril 2015, consultato il 18 septembre 2020. URL : http:// journals.openedition.org/studifrancesi/581; DOI : https://doi.org/10.4000/studifrancesi.581

Questo documento è stato generato automaticamente il 18 settembre 2020.

\section{(c) $(1) \&$}

Studi Francesi è distribuita con Licenza Creative Commons Attribuzione - Non commerciale - Non opere derivate 4.0 Internazionale. 


\title{
Manuela Raccanello, Proust in Italia. Le traduzioni della "Recherche"
}

\author{
Francesca Piselli
}

\section{NOTIZIA}

MANUELA RACCANELlo, Proust in Italia. Le traduzioni della "Recherche", Firenze, Le Lettere

Università, 2014, pp. 93.

1 Il contenuto ma denso volume di Manuela Raccanello figura a pieno titolo tra le pubblicazioni più importanti apparse in occasione del centenario della pubblicazione del primo libro della Recherche du temps perdu (1913) di Marcel Proust. Apprezzata traduttrice e studiosa nel campo sia della critica che della storia della traduzione, Raccanello analizza secondo un'ottica critico-comparativa le prime traduzioni italiane del capolavoro proustiano.

2 Il primo a dare conto ai lettori italiani di Du côté de chez Swann è stato Lucio D'Ambra, giornalista, romanziere e grande conoscitore della letteratura francese dell'Ottocento. In un breve articolo apparso nella «Rassegna Contemporanea» manifesta un aperto entusiasmo e una decisa ammirazione per l'opera proustiana, paragonando il suo autore a Stendhal. Si tratta tuttavia di una voce quasi isolata, dato che gli interventi critici successivi, tra cui quelli di Benedetto Croce, di Gabriele d'Annunzio e di Giuseppe Prezzolini, sono stati decisamente severi. Tra i pochi che hanno elogiato lo stile proustiano, vanno menzionati Giuseppe Ungaretti e Giacomo Debenedetti.

3 Il ritardo con cui si è avviato il processo traduttivo dell'opera proustiana è legato in primo luogo a questo clima polemico e in secondo luogo al fatto che l'impresa era innegabilmente impegnativa. Le condizioni sono maturate una decina di anni dopo, anche se occorre precisare che fino ai primi anni Quaranta del Novecento sono stati tradotti solo brevi passi della Recherche, tutti quanti pubblicati nelle riviste più attente alle novità letterarie. 
4 La prima traduzione, apparsa il 18 febbraio 1923 sulle colonne del quotidiano indipendente «Il Mondo», porta la firma di Corrado Alvaro. Il passo tradotto, intitolato La morte di Bergotte, appartiene al volume postumo La Prisonnière (1923). La versione dello scrittore calabrese risente della conoscenza limitata della Recherche e, come sottolinea Raccanello, la trama delle corrispondenze sonore si smaglia, le iterazioni lessicali e sintattiche dell'originale sono poco considerate (p. 11). Sotto il profilo lessicale si osserva una tendenza all'ennoblissement del lessico, laddove Proust selezionava quest'ultimo in funzione della precisione e non tanto dell'effetto.

5 Nonostante il regime fascista avesse vietato la traduzione dei romanzi dello scrittore francese perché potenzialmente sovversivi, negli anni 1924-1925 sono stati tradotti altri morceaux choisis, tra cui il passo intitolato I paesaggi di Elstir, pubblicato nell'«Esame» nel 1924 a opera di Renato Mucci e, sempre in quello stesso anno, Soggiorno a Venezia nel dopoguerra, ancora una volta sulle colonne de «Il Mondo», mentre l'anno successivo l'«Eco del Piave» ha ospitato la versione di un passo tratto da Sodome et Gomorrhe, tradotto dallo scrittore Giovanni Comisso.

Nel maggio del 1944 ha visto finalmente la luce una parte cospicua della Recherche. Il volumetto La precauzione inutile. Romanzo (Milano-Roma, Jandi Editori), ampia versione del romanzo La prisonnière siglata da Eugenio Giovannetti, ha inaugurato una nuova stagione editoriale. Un anno dopo appare Soggiorno a Venezia (Venezia, Edizioni del Cavallino), versione del terzo capitolo di Albertine disparue, firmata dal già ricordato Renato Mucci.

7 È stato con la fine della guerra che è scattata «l'ora $\mathrm{X}$, quella dell'attacco frontale al romanzo» come ha scritto Giacomo Debenedetti. Si è cominciato con la prima parte, ossia Du côté de chez Swann; risalgono al 1946, infatti, Casa Swann (Firenze, Sansoni), Un amore di Swann (Milano-Roma, Jandi-Sapi), La strada di Swann (Torino, Einaudi), tradotti rispettivamente da Bruno Schacherl, da Armando Landini e da Natalia Ginzburg.

8 Con quest'ultimo romanzo, La strada di Swann, Giulio Einaudi ha dato inizio a quell'ampio progetto editoriale, concretizzatosi nel 1951, che prevedeva la traduzione integrale della Recherche. Tale iniziativa ha coinvolto intellettuali e scrittori di spicco, tra cui Franco Calamandrei, traduttore, insieme a Nicoletta Neri, del romanzo All'ombra delle fanciulle in fiore (1949), Mario Bonfantini, che ha siglato I Guermantes (1949), Franco Fortini, traduttore di Albertine scomparsa (1951) e Giorgio Caproni, che ha curato la traduzione de Il tempo ritrovato (1951).

9 Particolarmente interessante è l'analisi condotta dall'A. nella seconda parte del suo lavoro. Con un'ammirevole finezza d'analisi, oltre che con un acuto senso critico, la studiosa si sofferma sulle traduzioni di Casa Swann di Bruno Schacherl e su La strada di Swann di Natalia Ginzburg.

10 La prassi traduttiva di Schacherl in Casa Swann si connota, per sua diretta ammissione, come un esercizio stilistico non sostenuto da riflessioni metodologiche, né tantomeno da preoccupazioni inerenti la ricezione testuale. Il risultato traduttivo risente dell'inesperienza del giovane intellettuale, all'epoca poco più che ventenne. Questi semplifica sistematicamente l'architettura sintattica, abolendo così la tensione intellettuale del testo di partenza, ridisegna i paragrafi, riformulando, scrive Raccanello, «la transizione da un periodo all'altro con un'interpunzione normativa» (p. 39). Ciò contribuisce con ogni evidenza a snaturare un libro che fa della mancanza dei 
dialoghi e dei capitoli - propri dei romanzi per così dire tradizionali - uno dei suoi tratti distintivi.

11 Nella ristampa del 1965, licenziata sempre da Sansoni, Casa Swann diventa Dalla parte di Swann, ma il traduttore, o forse l'editore, non sente la necessità di quella che Antoine Berman chiama retraduction. Ecco allora che ritroviamo la stessa dissoluzione della trama ipotattica di Casa Swann, senza tenere conto della lezione di Leo Spitzer, pur citato nell'Introduzione della versione del 1965, che aveva indicato nella complessità delle frasi proustiane, che tentano «per via sintattica una forma di pittura verbale» (p. 45), la peculiarità precipua della Recherche.

Al pari di Bruno Schacherl anche Natalia Ginzburg ha intrapreso il lavoro di traduzione del volume La strada di Swann a vent'anni, senza essersi mai accostata a questo tipo di lavoro, come rivela nella sua Nota del traduttore che accompagna la ristampa del 1990. Nel caso della Ginzburg non si riscontrano concessioni all'indole creativa e alla cifra stilistica personale. L'adesione alla struttura del testo di partenza è manifesta, arrivando talora, rileva Raccanello, ai limiti dell'interferenza linguistica (p. 70). La sua traduzione ha riscosso il plauso di Giacomo Debenedetti, nonostante il critico biellese vi avesse ravvisato alcune sviste, che non inficiavano comunque, a suo dire, il valore di un lavoro così impegnativo.

13 La casa editrice Einaudi ha fatto revisionare due volte la traduzione de La strada di Swann, affidandosi prima a Paolo Serini agli inizi degli anni Sessanta, e successivamente a Mariolina Bongiovanni Bertini nel 1978. Serini mantiene lo stesso titolo, ma opera numerose variazioni in nome di nuovi criteri traduttivi, applicati peraltro non sempre omogeneamente. L'intervento della Bertini, che recupera la forma originale di alcuni termini, ad esempio madeleine, che la Ginzburg aveva reso con «maddalenine», viene vissuto come abuso sul proprio testo da quest'ultima, come rivela nella Nota del traduttore apparsa nell'edizione de La strada di Swann licenziata da Einaudi nel 1990. La scrittrice ha considerato tale edizione come un gradito omaggio alla sua «vecchia, difettosa, appassionata» traduzione del 1946 (p. 87).

L'auspicio è che studi rigorosi di critica della traduzione, come quello presentato da Manuela Raccanello, siano condotti anche per le altre opere di Proust. 